\title{
TÌM HIỂU QUY TRÌNH ĐO VÀ XỬ LÝ SỐ LIỆ TRỌNG LỰC TUYỆT ĐỐI BẰNG MÁY FG5x - 245
}

\author{
NGUYẼ̃N PHI SƠN, NGUYẼN TUÂN ANH \\ Viện Khoa học Đo đạc và Bản đồ
}

\section{Tóm tắt:}

Trọng lực là một chuyên ngành khoa học về trái đất, nghiên cứu xác định lực hút của trái đất và sự biến thiên của nó theo thời gian. Trọng lực có ý nghĩa khoa học và thực tiễn to lớn, áp dụng cho những ngành khoa học cơ bản như: trắc địa - bản đồ, địa chất - khoáng sản, tài nguyên nước, hải dương học, thiên văn học, hàng không, vật lý trái đất, quốc phòng..., trong số các chuyên ngành này có những nhiệm vụ đòi hỏi phép đo trọng lực với độ chính xác rất cao cỡ một vài microGal, nhu: xây dựng hoặc đo lặp mạng lưới trọng lực cơ sở, trọng lực hạng I quốc gia, thăm dò trọng lực các tỷ lệ rất lớn 1/500 đến 1/5000, xác định biến thiên mực nước ngầm ... Cho đến năm 2012, Việt Nam chưa có máy đo trọng lực tuyệt đối mà phải hợp tác với Liên bang Nga trong xây dụnng và hoàn thiện hệ thống trọng lực quốc gia. Trước yêu cầu của lĩnh vực đo đạc và bản đồ trong xây dựng mô hình Quasigeoid, hoàn thiện hệ độ cao hiện đại... cũng như yêu cầu ứng dụng trong các chuyên ngành khác, Viện Khoa học Đo đạc và Bản đồ đã được Bộ Tài nguyên và Môi trường tăng cường năng lực nghiên cứu về trắc địa cao cấp với các máy đo trọng lực thế hệ mới nhất, trong đó có hệ thống máy đo trọng lực tuyệt đối FG5x. Đây là hệ thống thiết bị hiện đại và chính xác hàng đầu thế giới hiện nay do Mỹ sản xuất. Trong 2 năm qua các cán bộ của Viện Khoa học Đo đạc và Bản đồ đã nghiên cứu khai thác sử dụng, ứng dụng trong công tác trắc địa bản đồ, làm chủ được hệ thống, đưa ra các quy trình đo đạc, xử lý số liệu, đặc biệt là đã nghiên cứu ứng dụng trong thăm dò trọng lực về khoáng sản rắn, ứng dụng trong nghiên cứu động thái nước dưới đất. Trong bài báo này xin giới thiệu về máy FG5x bao gồm tính năng, quy trình đo và xử lý dữ liệu đo trọng lực tuyệt đối.

\section{Giới thiệu về máy đo trọng lực tuyệt đối FG5x và khả năng ứng dụng}

Năm 2012, Viện Khoa học Đo đạc và Bản đồ được Bộ Tài nguyên và Môi trường tăng cường năng lực nghiên cứu với các máy đo trọng lực mà cho đến nay ở Việt Nam chưa có, nhằm phát triển các ứng dụng và thực hiện các dự án điều tra cơ bản về trọng lực trên lãnh thổ Việt Nam, hợp tác thực hiện trên lãnh thổ Lào, Campuchia. Dự án đầu tư với 2 bộ thiết bị quan trọng là hệ thống đo trọng lực tuyệt đối FG5x số hiệu
245 và hệ thống đo trọng lực hàng không AIR TAGS6. Bộ máy TAGS6 phục vụ đo trọng lực chi tiết cho vùng núi cao, biên giới chưa có dữ liệu trọng lực (chiếm gần $1 / 3$ diện tích lãnh thổ Việt Nam). Còn FG5x phục vụ đo thu nhận dữ liệu trọng lực tuyệt đối với độ chính xác rất cao, như các mốc trọng lực cơ sở, hạng I và các ứng dụng khác. FG5x hoạt động dựa trên nguyên lý giám sát vật rợ tự do trong buồng chân không để xác định vị trí của quả rơi bằng một hàm số thời gian. Các quỹ đạo rơi tự do được tham chiếu đến một hệ thống hoạt

Ngày nhận bài: 21/3/2017, ngày chuyển phản biện: 22/3/2017, ngày chấp nhận phản biện: 28/3/2017, ngày chấp nhận đăng: 31/3/2017 
động rất ổn định gọi là Superspring. Superspring được cách ly các tác động địa chấn. Quá trình rơi được giám sát rất chính xác bằng một hệ thống giao thoa LaserHeNe và đồng hồ nguyên tử rubidium.

Các thành phần của hệ thống FG5x: gồm 4 hệ thống con, hệ thống buồng rơi; hệ thống Laser (WEO100); hệ thống điều khiển (Controler); hệ thống máy tính kiểm soát và phần mềm thu nhận xử lý dữ liệu.

Thông số kỹ thuật của hệ thống FG5x: tổng trọng lượng $320 \mathrm{~kg}$ trong 6 hộp; tổng khối lượng 1,5m³ phạm vi không gian yêu cầu $3 \mathrm{~m}^{2}$; điện áp đầu vào 110-240 VAC (switchable), 50 - 60Hz; công xuất: $500 \mathrm{~W}$.

Hiệu xuất kỹ thuật của FG5x: độ chính xác $1-2 \mu \mathrm{Gal}$; phạm vi hoạt động: WorldWide; nhiệt độ hoạt động $10^{\circ} \mathrm{C}$ đến $30^{\circ} \mathrm{C}$; độ ẩm nhỏ hơn $60 \%$.

Về các ứng dụng của FG5x: với độ chính xác rất cao của $F G 5 x$ có thể phân biệt được sự biến thiên của trường trọng lực trái đất ở mức độ $\pm 2 \mu \mathrm{Gal}$ trong khoảng thời gian đo 6,25 giờ. Phần mềm xử lý dữ liệu đo " $g 9$ " đã được tích hợp các mô hình cải chính, đảm bảo loại trừ các hiệu ứng cho các nhu cầu ứng dụng khác nhau.

Sau đây xin giới thiệu một số ứng dụng cơ bản của đo trọng lực tuyệt đối độ chính xác cao mà hệ thống FG5x có khả năng đáp ứng:

- Xây dựng các mạng lưới trọng lực quốc gia các cấp hạng với yêu cầu độ chính xác về gia tốc lực trọng trường từ $\pm 5 \mu \mathrm{Gal}$ đến $\pm 20 \mu \mathrm{Gal}$;

- Xác định sự biến thiên cục bộ của trường trọng lực theo thời gian với độ chính xác cao hơn $\pm 5 \mu \mathrm{Gal}$, thậm chí đến \pm
$0,1 \mu \mathrm{Gal}$.

- Xây dựng mạng lưới tựa trọng lực phục vụ thăm dò vi trọng lực ở các tỷ lệ $1 / 500$, 1/1000, 1/2000, 1/5000; Nghiên cứu khảo cổ [3].

- Điều tra nước ngầm và đánh giá sự thay đổi của mực nước ngầm. Sự biến thiên theo mùa của mực nước ngầm bằng các phép đo trọng lực theo thời gian. Xác định độ ẩm đất [5].

- Giám sát mực nước biển toàn cầu và giám sát sự nóng lên toàn cầu từ phép đo ít nhất một chu kỳ triều nhật đầy đủ [5].

- Nghiên cứu đánh giá áp lực của khí quyển lên bề mặt trái đất và độ cao của sóng thủy triều (ảnh hưởng của áp lực khí quyển lên bề mặt trái đất ở mức $0,36 \mu \mathrm{Gal} / 1$ millibar. Sự biến thiên của trọng trường ở vùng ven biển do các sóng thủy triều đạt tới mức $0,2 \mu \mathrm{Gal}$ khi sóng thủy triều thay đổi 1 $\mathrm{cm})$ [2].

\section{Quy trình đo trọng lực tuyệt đối bằng $\mathrm{FG} 5 \mathrm{x}$}

\subsection{Công tác chuẩn bị}

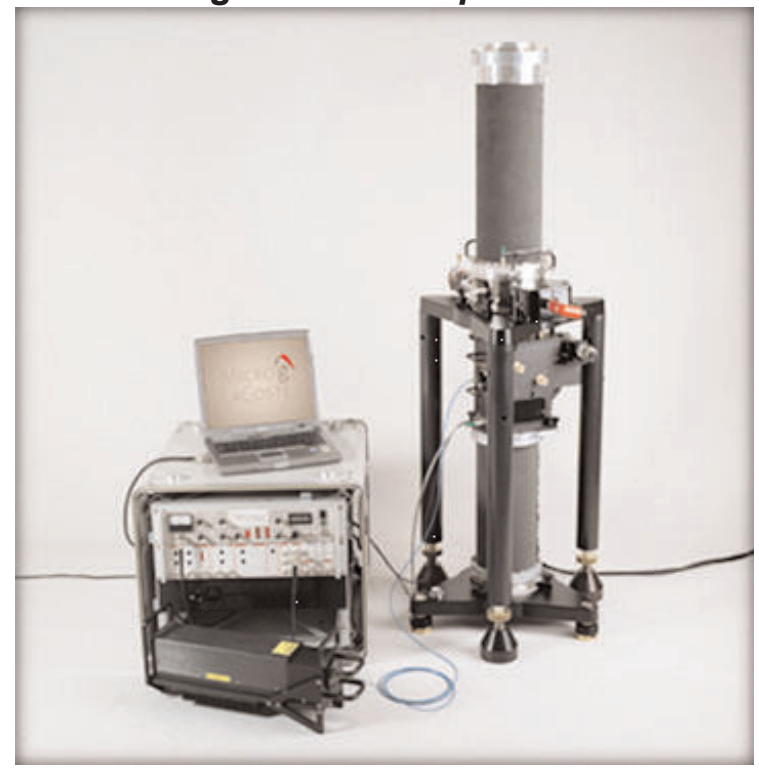


Chuẩn bị các thiết bị đồng bộ của hệ thống FG5x (kèm theo buồng bảo ôn đảm bảo cho hệ thống FG5x luôn ở trạng thái nhiệt độ thấp hơn $30^{\circ} \mathrm{C}$, độ ẩm thấp hơn $85 \%$ ), danh mục thiết bị kèm theo đầy đủ và trong trạng thái hoạt động tốt. Máy phát điện dự phòng.

Chuẩn bị máy đo trọng lực tương đối để đo gradient đứng tại điểm cần đo (loại ZLS).

Chuẩn bị máy đo tọa độ (GPS), độ cao thủy chuẩn.

Vận chuyển thiết bị đến điểm đo bằng ô tô chuyên dùng, có hệ thống giảm xóc; Yêu cầu không gian quanh điểm đo phải đặt được buồng bảo ôn khoảng $6 \mathrm{~m}^{2}$.

\subsection{Lắp đặt máy trọng lực FG5x}

- Lắp đặt giá đỡ của FG5x: theo trình tự của catalog của nhà sản xuất. Đo chiều cao của giá đỡ so với mặt mốc bằng dụng cụ theo máy và ghi lại giá trị đo được.

- Lắp đặt thiết bị điện: kiểm tra lại các thiết lập điện áp đầu vào phù hợp với dòng điện áp AC, từ 100-240V AC, 50-60HZ.

- Bơm ion đã được duy trì cho buồng rợ chân không bằng nguồn điện từ pin, gắn thêm nguồn điện $\mathrm{AC}$ để cung cấp cho máy bơn ion.

- Các thao tác ngắt, bật nguồn điện $\mathrm{AC}$ giữa các hệ thống con: WEO100, FG5x, Controler Hight Voltge theo catalog của nhà sản xuất.

- Toàn bộ hệ thống phải bật trước khi đo ít nhất là $1 \mathrm{~h}$ đồng hồ để cho bộ phát laser đạt đến trạng thái cân bằng nhiệt.

- Kiểm tra và căn chỉnh: Kiểm tra chùm tia laser thẳng đứng; Chỉnh vân giao thoa (Fring Optimization); Điều chỉnh Twiddler and Lower cho đến khi tia kiểm tra (test beam) và tia tham chiếu (reference beam) chồng lên nhau; Kết nối ANALOG FRINGE trên SIM đến Oscilloscope, với thông số (Tỷ lệ dọc $=50 \mathrm{mV} /$ div; Tỷ lệ ngang $=2 \mu \mathrm{sec}$ /div; Nguồn đầu vào; Tự động kích hoạt (automatic trigger) tại OV; Chỉnh các tín hiệu vân trên Osillocope bằng cách điều chỉnh gương \# 2.

- Các lưu ý: Không vận hành hệ thống máy FG5 nếu vân giao thoa cực đại dưới $300 \mathrm{mV}$ hoặc cao hơn $400 \mathrm{mV}$. Điều này có thể ảnh hưởng xấu đến giá trị đo trọng lực; Ngừng chế độ OSC bằng cách nhấn nút DROP trên SIM. Điều này mang lại sự an toàn cho vật rơi vào cuối của chu kỳ OSC tiếp theo. Nhấn TRIG sẽ đặt chu trình rơi ở chế độ nghỉ (REST) vào cuối của chu kỳ OSC tiếp theo. Nhấn RESET lại sẽ ngừng tất cả lực đang có để thả vật rơi từ bất cứ vị trí nào; Khi năng lượng laser lên nằm ngoài phạm vi, kiểm tra điện áp DC (nguồn điện bộ phát laser) trên bộ điều khiển WEO (thường 5- $10 \mathrm{~V}$ là đủ); Kiểm tra lại độ thẳng đứng của tia laser bằng lọ chất lỏng lần thứ 2.

\subsection{Quá trình đo}

Thông qua phần mềm " $g 9$ " để khai báo các thông số:

- Về điểm đo: tên điểm, mã điểm, tọa độ, độ cao, gradient đứng của điểm, độ cao máy (tổng 2 độ cao đo được khi ta lắp đặt máy FG5x), độ lệch trục quay của trái đất (lấy ở trang Web của hãng www.microglacoste.com và cập nhật 1 tuần 1 lần), độ cao chuyển đổi (Transfer Height) đặt $=0$;

- Về hệ thống máy: lựa chọn loại tần số của laser dạng $D, E, F, G$ bằng đồng hồ đo 
dòng điện. Kết nối đồng hồ đo dòng với OUTPUT, lần lượt đo dòng ở các vị trí $\mathrm{D}, \mathrm{E}$, F, G và cập nhật vào "WEO 100 laser Setup" phần $(\mathrm{V})$;

- Về tần suất một lần đo: Số lượng SETđo (thường đặt 12 sets cho một điểm). Số lần quả rơi trong một lầnđo Drops/Set (thường thường ta đặt 100 lần). Khoảng thời gian giữa 2 lần đo (thường đặt là 60 phút). Thời gian giữa 2 lần quả rơi rơi (thường đặt là 5 giây).

- Thông tin về điều khiển, thông tin về người đo.

- Tiến hành đo, dữ liệu mỗi điểm đo được lưu thành project.txt.

\subsection{Kết thúc đo và tháo máy}

- Khi kết thúc đo cần sao lưu dữ liệu ra một bộ nhớ khác.

- Tắt nguồn điện ở SIMx (Đưa POWER về chế độ OFF ở SIMx), tắt nguồn điện cao áp (vặn chìa khóa), tắt nguồn điện ở bộ phát laser, tắt nguồn chính $\mathrm{AC}$ (không được tắt nguồn của bơm ion ngoại trừ việc vận chuyển đi xa).

Khóa buồng lò xo bằng cách kéo núm khóa ra và xoay khóa theo chiều kim đồng hồ $180^{\circ}$ để khóa lại.

Tháo tất cả các dây cáp đã được kết nối khi lắp đặt máy và cất vào túi đựng dây cáp.

Tháo buồng rơi; tháo chân buồng rơi

Tháo buồng giao thoa ra khỏi buồng lò xo và cho vào hộp.

- Buồng lò xo phải thực sự được khóa, đóng nắp chống bụi ở trên đỉnh, tháo buồng lò xo ra khỏi giá đỡ và đưa toàn bộ vào thùng.

\section{Xử lý số liệu bằng phần mềm " $g 9$ "}

Phần mềm " $g 9$ " đồng bộ cùng hệ thống FG5x hoạt động trong môi trường Windows $^{\mathrm{TM}}$. $g 9$ được sử dụng với tất cả các máy đo trọng lực tuyệt đối $M G L$ của hãng Micro g Lacoste, bao gồm cả các FG-5, A10, FG-L. Phần mềm " $g 9$ " được xây dựng dựa trên các thuật toán "Time Interval Analyzer (TIA)" để ghi lại các thông tin liên quan đến việc kiểm soát thời gian chính xác của quả rơi (khoảng cách rơi là một hàm của thời gian). Công thức chính xác là:

$$
\left.\begin{array}{rl}
x_{i}=x_{0}+v_{0} \widetilde{t}_{i}+\frac{g_{0} \widetilde{t}_{i}^{2}}{2}+\frac{\alpha_{0} \tilde{t}_{i}^{2}}{2}+\frac{1}{6} \gamma_{0}{\widetilde{t_{i}}}^{3}+\frac{1}{24} \gamma g_{0}{\widetilde{t_{i}}}^{4} \\
\tilde{t}=t_{i}-\frac{\left(x_{i}-x_{0}\right)}{c}
\end{array}\right\}
$$

Việc xử lý cải chính đối với số liệu trọng lực toàn phần được "g9" tính trực tiếp gồm:

- Cải chính triều đại dương (Ocean Load).

- Cải chính áp xuất khí quyển (Barometric Pressure).

- Cải chính triều trái đất (Earth Tide ETGTAB).

- Cải chính chuyển động cực của trái đất (Polar Motion).

- Cải chính chuyển đổi độ cao (Transfer Height).

- Cải chính tham chiếu (Reference Xo).

Ngoài các tính toán và điều chỉnh trên, phần mềm cung cấp khả năng xử lý thời gian thực và thống kê cho người sử dụng biết chi tiết về các tham số khác như:

- Thông tin về trạm đo: tọa độ, độ cao, nhệt độ, áp xuất, gradient đứng...

- Thông tin hệ thống về FG5x: thông số 


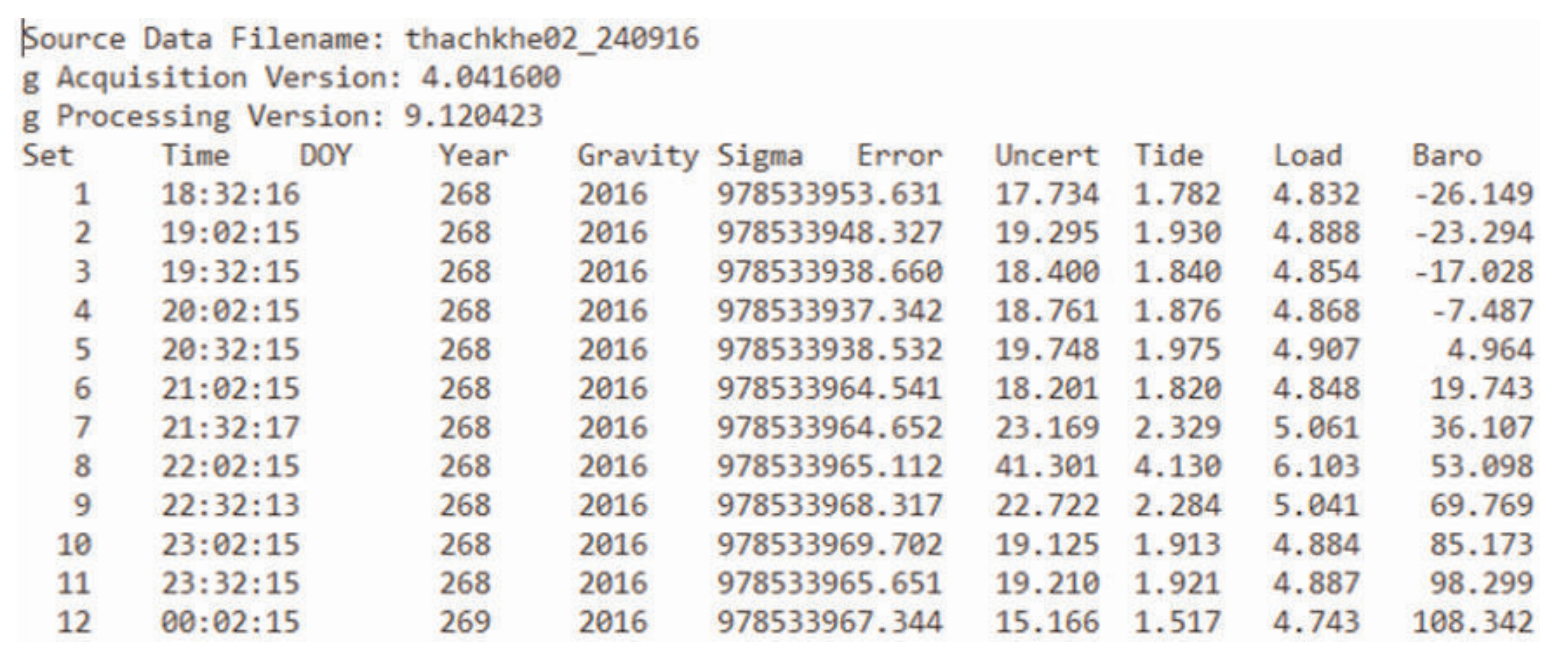

về các thiết bị đồng bộ về tần số, lazer...

- Thông tin về tiêu chuẩn thu nhận dữ liệu đo: yêu cầu về các SET đo, thời gian, độ chính xác, các yêu cầu số cải chính, thông tin về điều khiển, thông tin về người đo...

Mỗi ca đo thường phải kéo dài trong nhiều SET đo, mỗi SET đo là chuỗi thông số đảm bảo cho tính toán một trị trọng lực toàn phần và xác định kèm theo các hiệu ứng tại thời điểm đo. Các thông số setup cho mỗi SET đo được đặt tùy thuộc vào từng yêu cầu nhiệm vụ.

Kết quả tính toán, xử lý bằng phần mềm "g9" được xuất ra ở dạng file .txt, bao gồm các cột dữ liệu về mỗi SET đo như: thời gian đo, giá trị trọng lực toàn phần, các giá trị cải chính (triều đại dương, triều cứng trái đất, mặt trăng, mặt trời, vĩ độ, độ cao....). Tùy thuộc vào mục đích sử dụng giá trị trọng lực tuyệt đối, người sử dụng sẽ tính cải chính vào giá trị trọng lực toàn phần (Gravity). (xem bảng)

\section{Kết luận}

So với các hệ thống đo tuyệt đối trên thế giới hiện nay, FG5x là thiết bị hiện đại, đảm bảo độ chính xác cao và độ ổn định nhất. Các nước tiên tiến trên thế giới, đặc biệt là nhiều nước Châu Âu đã và đang sử dụng FG5x trong đo trọng lực tuyệt đối nhiều vùng lãnh thổ. Tuy nhiên FG5x còn hạn chế ở việc chưa thích nghi với khí hậu nóng ẩm ở Việt $\mathrm{Nam}$, vấn đề này đã được khắc phục bằng việc chế tạo thêm một buồng bảo ôn, nhằm giữ các điều kiện tốt nhất về nhiệt độ và độ ẩm trong suốt thời gian đo ở thực địa. Trong thời gian qua, các cán bộ kỹ thuật của Viện đã tiến hành đo bằng $F G 5 x$ ở nhiều vùng ở Việt Nam, đã đúc kết thành quy trình đo, sử dụng và bảo quản máy, cho đến nay đã làm chủ được công nghệ. Với khả năng ứng dụng trong nhiều lĩnh vực, cho thấy tiềm năng của $F G 5 x$ là rất lớn. Bên cạnh nhiệm vụ chính là đo lặp hệ thống trọng lực cơ sở quốc gia, hạng I quốc gia của Việt Nam, trong thời gian tới $F G 5 x$ sẽ tham gia đo trọng lực cở sở và trọng lực hạng I cho Vương quốc Campuchia. Đây là lần đầu tiên, Việt Nam có bộ thiết bị đo trọng lực tuyệt đối hiện đại, việc nghiên cứu tìm hiểu, vận hành và đặc biệt là phát triển các ứng dụng không những trong lĩnh vực đo đạc bản đồ mà còn trong các lĩnh vực khác, như 
địa chất khoáng sản, tài nguyên nước... là hết sức cần thiết. Năm 2016, Viện Khoa học Đo đạc và Bản đồ đã hoàn thành đề tài "Nghiên cứu ứng dụng đo trọng lực bằng máy $\mathrm{FG} 5 \mathrm{x}$ phục vụ điều tra, đánh giá nước dưới đất và một số khoáng sản rắn ở Việt Nam" mã số TNMT 07.42, bài báo được viết từ kết quả thực hiện đề tài cấp Bộ nói trên. $O$

\section{Tài liệu tham khảo}

[1]. Tôn Tích Ái. 2003. "Trọng lực và thăm dò trọng lực". NXB Đại học Quốc gia Hà Nội.

[2]. Hà Minh Hòa. 2014. 'Lý thuyết và thực tiễn của trọng lực trắc địa". NXB Khoa học và Kỹ thuật.

[3]. Micro g Lacoste. User's Manual 2012.

[4]. Micro g Lacoste. 2012. "g9 Absolute Gravity Data Acquisition and Processing Software". www.microglacoste,com.

[5]. Trần Văn Nhạc. 2008. "Phương pháp trọng lực trong địa vật lý". NXB Khoa học và Kỹ thuật. $O$

\section{Summary}

\section{Understanding and treatment process measuring gravity data with absolutely FG5x machine - 245}

Nguyen Phi Son, Nguyen Tuan Anh

Institute of Geodesy and Cartography

Gravity is a science of the earth, researchers determined Earth's gravity and its variation over time. Gravity has scientific significance and tremendous practical, applied to the walls such as basic sciences: mapping surveying, geology, water resources, oceanography, astronomy, aeronautics, earth physics, defense..., among the majors has the task requires gravity measurements with very high accuracy within several microGal when building or network repeater gravity measurement basis, Grade I national gravity, gravity exploration rate of $1 / 500$ to $1 / 5000$ huge, determine groundwater level variation... for 2012 Vietnam counted no absolute gravity meter that must cooperate with Russian Federation in developing and improving the system of national gravity. Before the requirements of the field of geodesy and cartography, as well as key applications in other disciplines, the Institute is the Ministry of Natural Resources and Environment KHDDBD strengthening research on advanced geodetic gravity meters with the latest generation, including the system of absolute gravity meter FG5x, this is the system of modern equipment and the most accurate in the world today by the US producer. In 2 years the scientific staff of the Institute of surveying and mapping has researched utilization, applications in mapping surveying work, to master the system, making the process of measuring, processing data, particularly in applied research has explored the gravity of solid minerals, research applications in groundwater dynamics. In this paper would like to introduce FG5x machines include features and process measurement data processing and measurement of absolute gravity. $O$ 